\title{
Efficacy of continuous positive airway pressure treatment in treating obstructive sleep apnea hypopnea syndrome associated with carotid arteriosclerosis
}

\author{
YONG-QIAN JIANG，JIN-SHAN XUE，JUAN XU，ZHI-XIANG ZHOU and YOU-LIN JI \\ Department of Respiratory Medicine, The First People's Hospital of Yancheng, Yancheng, Jiangsu 224006, P.R. China
}

Received September 27, 2016; Accepted May 15, 2017

DOI: $10.3892 /$ etm.2017.5308

\begin{abstract}
Sleep apnea negatively impacts patients' ability to oxygenate the bloodstream during sleep and has far-reaching, deleterious effects. The present study sought to assess the correlation between obstructive sleep apnea hypopnea syndrome (OSAHS), carotid atherosclerosis, and blood pressure variability (BPV), and to evaluate the therapeutic effects of continuous positive airway pressure (CPAP). Patients with OSAHS were classified as mild, moderate, or severe according to their condition and compared with healthy control participants. CPAP treatment was used to treat patients with OSAHS for 6 months. Prior to CPAP treatment, the apnea-hypopnea index (AHI), lowest blood oxygen saturation $\left(\mathrm{LSaO}_{2}\right)$, carotid intima media thickness (IMT), and plasma levels of endothelin-1 (ET-1), nitric oxide (NO), and tumor necrosis factor- $\alpha$ $(\mathrm{TNF}-\alpha)$ were measured in all participants, along with the low frequency components of BPV (BPV LF). The results demonstrated that carotid IMT, AHI, plasma ET-1, and plasma TNF- $\alpha$ were significantly higher in patients with OSAHS than those in the control group $(\mathrm{P}<0.05)$; whereas $\mathrm{LSaO}_{2}$ and plasma NO levels were significantly higher in the control group $(\mathrm{P}<0.05)$. The degree to which these indices differed was associated with the severity of OSAHS. Furthermore, the carotid IMT of patients with OSAHS was significantly correlated with $\mathrm{AHI}(\mathrm{P}=0.037)$, plasma ET-1 $(\mathrm{P}=0.001)$, plasma NO $(\mathrm{P}<0.001)$, BPV LF before retiring $(\mathrm{P}<0.001)$. Following CPAP treatment, the observation indices of patients with moderate or severe OSAHS improved significantly $(\mathrm{P}<0.01)$. These results support the use of CPAP to improve the significant vascular endothelial dysfunction, increased inflammatory response, and high blood pressure variability correlated with carotid atherosclerosis observed in patients with OSAHS.
\end{abstract}

Correspondence to: Professor You-Lin Ji, Department of Respiratory Medicine, The First People's Hospital of Yancheng, 14 Yuehe Road, Yancheng, Jiangsu 224006, P.R. China E-mail: ycjiangyq@126.com

Key words: obstructive sleep apnea hypopnea syndrome, carotid atherosclerosis, blood pressure variability, continuous positive airway pressure ventilation, therapeutic effects observation

\section{Introduction}

Sleep apnea is the brief, involuntary cessation of breathing during sleep. Sufferers often experience loud, sudden snoring and sudden body movements as a result. Sleep apnea is predominantly often caused by obstruction of the upper airway and obstructive sleep apnea hypopnea syndrome (OSAHS) refers to a collection of physio-pathological syndromes associated with sleep apnea, including body hypoxemia and hypercapnia. Primary clinical manifestations of OSAHS are apnea and hypopnea, snoring, sleep structure disorder, and lethargy during waking hour (1). The morbidity rate of OSAHS among adult males is $\sim 4 \%$, whereas the morbidity rate among adult females is $\sim 2 \%$. The morbidity rate among people aged 60 and above may be as high as $20-40 \%$ (2).

Obesity, age, and muscular flaccidity have been demonstrated to be risk factors for OSAHS, which is thought to have genetic origins in genes associated with obesity, metabolic syndrome, craniofacial structural abnormalities, defects in the muscular control of ventilation and the upper airway, and sleep and circadian rhythm disorders (3). In particular, abnormalities in blood lipid distribution genes, such as leptin, insulin-like growth factor-1, glucokinase, adenine nucleotide deaminase, melatonin-3 receptor, glucose-regulating protein, TNF- $\alpha$ and $\beta$, adrenergic receptor, orexin, and sugar regulating proteins, have been associated with OSAHS, as well as receptor protein tyrosine kinase, nerve growth factor, endothelin-1 (ET-1) and -3, nitric oxide synthase, and angiotensin converting enzyme (4). OSAHS has also been found to be associated with hypertension, insulin resistance, type 2 diabetes, arrhythmia, heart failure, cerebrovascular disease, ocular lesions, hypertension, coronary heart disease, and cerebral stroke (5). Chronic intermittent hypoxia brought on by OSAHS can lead to reperfusion injuries to the heart and cause oxidative stress reactions, lipid metabolism disorders, abnormal sympathetic nerve activation, and vascular endothelial functional disorder (6).

Abnormal circulating levels of inflammatory factors resulting from OSAHS are thought to contribute to the development of atherosclerosis (7), thus the prevention of OSAHS-associated inflammatory reactions is of notable clinical interest. High expression of an atherosclerosis-related protein, endothelin-1 (ET-1), has been detected in the 
peripheral blood of patients with OSAHS (8). ET-1, which is a potent vasoconstrictor, has been demonstrated to be correlated with the severity of OSAHS and continuous positive airway pressure (CPAP) treatment has been shown to be effective in lowering circulating ET-1 (9).

Nitric oxide (NO) has an important role in blood pressure and immune system regulation and its dysregulation is associated with cardiac failure, hypertension, coronary heart disease, diabetes, and hyperlipidemia (10). Patients with OSAHS have decreased circulating NO levels, suggesting that NO may also be involved with OSAHS-related cardiovascular and cerebrovascular diseases (10). Effective CPAP treatment has been demonstrated to reduce the occurrence of OSAHS-related cardiovascular distress by improving NO levels (11). Patients with OSAHS also have a high expression of TNF- $\alpha$ in peripheral blood and the independent application of CPAP and postoperative application of CPAP sequential treatment have been shown to lower the concentration of TNF- $\alpha$ and other indicators of hypoxemia $(12,13)$.

Patients with OSAHS often experience abnormal blood pressure variations, which are thought to be a result of nocturnal hypoxemia (14). After controlling for age, body mass index (BMI), and blood pressure, the apnea-hypopnea index (AHI) of patients with OSAHS is positively correlated with 24-h systolic and diastolic pressure variability, daytime systolic and diastolic pressure variability, and nocturnal systolic and diastolic pressure variability (15). OSAHS can further aggravate blood pressure elevation in patients with cardiovascular and cerebrovascular diseases and destroy the circadian rhythm of blood pressure (16). Effective CPAP, along with drug therapy, has been shown to improve the circadian rhythm of blood pressure and lower the category and dosage of combined blood pressure lowering agents, improving prognosis $(17,18)$.

Surgical treatment to correct OSAHS is highly invasive and has poor long-term efficacy. CPAP has the advantage of being non-invasive and is simple to operate, making it the preferred treatment for OSAHS (19). The present study aimed to assess the correlation between OSAHS, carotid atherosclerosis, and blood pressure variability (BPV), and to evaluate the therapeutic effects of CPAP in order to inform ongoing efforts to improve CPAP treatments and reduce the risk of cardiovascular disease in patients with OSAHS.

\section{Materials and methods}

Study participants. In total, 120 patients with OSAHS that visited the Sleep Center of the First People's Hospital of Yancheng City between June 2013 and June 2015 were evaluated for participation in the present study. All patients conformed to the diagnostic criteria presented in the Guideline for OSAHS Diagnosis and Treatment (Modified Version) formulated by the Sleep Breathing Disorder Study Group of China, Society of Respiratory Disease Study of Chinese Medical Association (20), and diagnosis was confirmed by polysomnogram. None of the patients received surgical or mechanical ventilation treatment before inclusion in the study. Patients with heart disease, malignant tumors, serious hepatic or renal functional lesions, or chronic inflammatory diseases were excluded, along with any patients with a history of serious lung diseases, hyperlipemia, diabetes, hypertension, a history of serious trauma within 2 weeks of enrollment, or long-term application of anti-inflammatory drugs, anticoagulant drugs, or drugs that may influence the observation indices used in the present study. Of the patients included in the study, 105 were men and 15 were women, ranging in age from 24 to 67 years with a mean age of $46.4 \pm 10.8$ years. Mean BMI was $28.2 \pm 4.3 \mathrm{~kg} / \mathrm{m}^{2}$.

Patients were assigned into groups according to their AHI: a mild group (15 times/h>AHI $\geq 5$ times/h; 28 patients), moderate group (30 times/h>AHI $\geq 15$ times/h; 48 patients), and severe group (AHI $>30$ times/h; 44 patients). A total of 40 healthy volunteers made up the control group and were matched with the patients in the case group in terms of age, sex, and BMI (Table I). OSAHS was ruled out for the control group after clinical examination, and the same exclusion criteria were applied. The study protocol was approved by the Medical Ethics Committee of the First People's Hospital of Yancheng City (Yancheng, China), and all subjects provided written informed consent.

Experimental treatment. Patients in the moderate and severe OSAHS groups underwent 6 months of CPAP treatment. On the first night of treatment, a breathing machine was used to perform CPAP pressure titration with the initial pressure set as $4 \mathrm{~cm} \mathrm{H}_{2} \mathrm{O}$. The pressure was subsequently adjusted according to the patient's apnea-hypopnea status every $5 \mathrm{~min}$ until apnea disappeared during each sleep period in all body positions and the percentage of arterial oxygen saturation $\left(\mathrm{SaO}_{2}\right)$ was $>90 \%$. At this point, the CPAP pressure was determined to be the optimal ventilation pressure and CPAP treatment was administered at this pressure $\left(8-15 \mathrm{~cm} \mathrm{H}_{2} \mathrm{O}\right)$ continuously for 5 to $8 \mathrm{~h}$ whilst patients slept each evening for 6 months.

Cardiovascular health indicators and detection methods. All indicators were determined at enrollment. A multi-lead sleep monitor was used to determine AHI and lowest $\mathrm{SaO}_{2}$ $\left(\mathrm{LSaO}_{2}\right)$ values. A color Doppler was used to determine carotid intima-media thickness (IMT) by a senior physician in the Ultrasound Department. With the probe frequency set at $8 \mathrm{MHz}$, the posterior wall at the distal end close to the furcation of the common carotid artery and the site $1 \mathrm{~cm}$ above the initial part of internal carotid artery were measured three times and the mean was calculated. Morning fasting peripheral vein blood draws were collected and, following anticoagulation, plasma was stored at $-80^{\circ} \mathrm{C}$ for subsequent testing. ELISA was used to detect NO (cat. no. 191426; BioAssay Systems LLC; Hayward, CA, USA) and TNF- $\alpha$ (cat. no. EK0526; Wuhan Boster Biological Technology, Ltd., Wuhan, China) according to the manufacturer's protocol and plasma ET-1 was detected by radioimmunoassay. Low frequency blood pressure variability (BPV LF) was measured via lectrocardiogram (ECG) $5 \mathrm{~min}$ before and $5 \mathrm{~min}$ after sleeping.

Statistical analysis. The SPSS 20.0 statistical package (IBM Corp, Armonk, NY, USA) was used to establish a database from which statistical analysis was performed. Measurement data were expressed as the mean \pm standard deviation. Single 
Table I. Baseline characteristics of the study participants.

\begin{tabular}{lcccc}
\hline Indices & Mild group $(\mathrm{n}=28)$ & Moderate group $(\mathrm{n}=48)$ & Severe group $(\mathrm{n}=44)$ & Control group $(\mathrm{n}=40)$ \\
\hline AHI (time/h) & $9.18 \pm 2.57$ & $21.29 \pm 4.02$ & $39.52 \pm 6.41$ & $2.13 \pm 1.26$ \\
Age (years) & $46.1 \pm 9.5$ & $46.5 \pm 10.9$ & $47.1 \pm 11.2$ & $46.5 \pm 12.3$ \\
Sex (male/female) & $25 / 3$ & $41 / 7$ & $39 / 5$ & $35 / 5$ \\
BMI $\left(\mathrm{kg} / \mathrm{m}^{2}\right)$ & $27.8 \pm 4.6$ & $28.8 \pm 5.1$ & $28.6 \pm 5.5$ & $27.5 \pm 6.2$
\end{tabular}

BMI, body mass index.

A



C

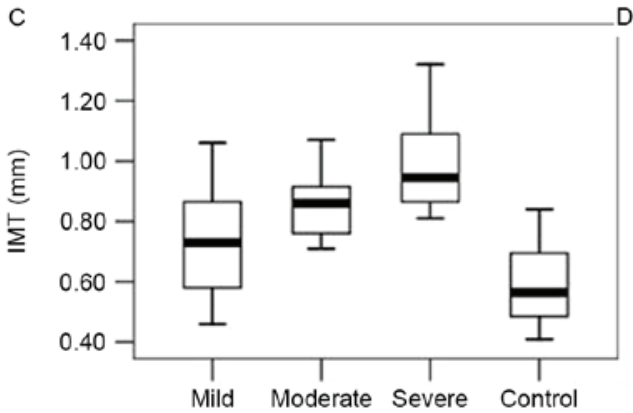

$\mathrm{E}$
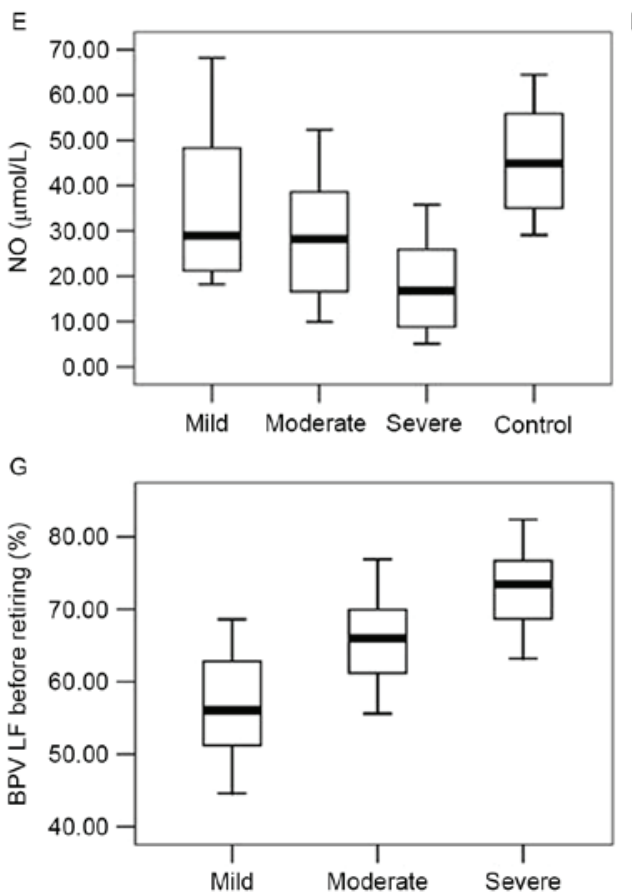

B
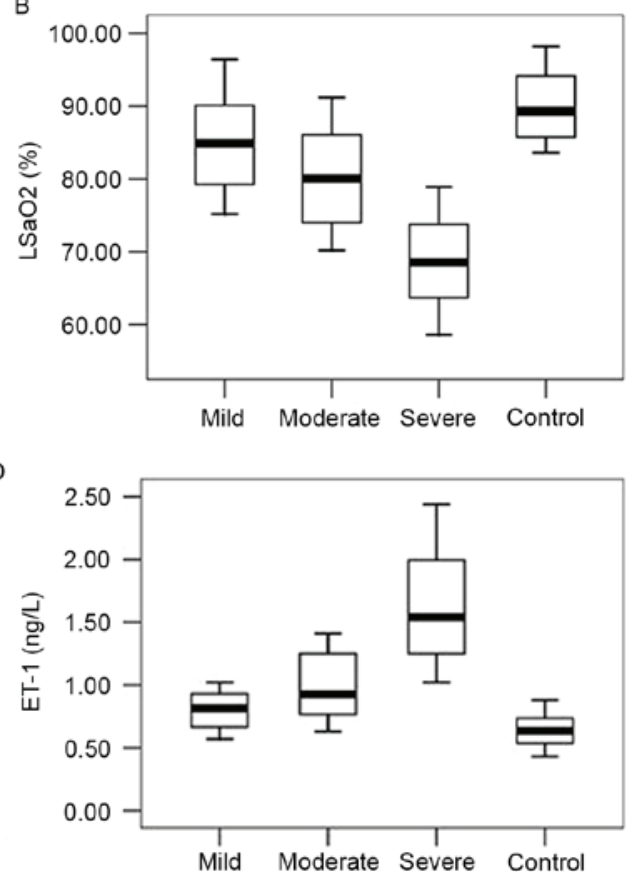

$\mathrm{F}$
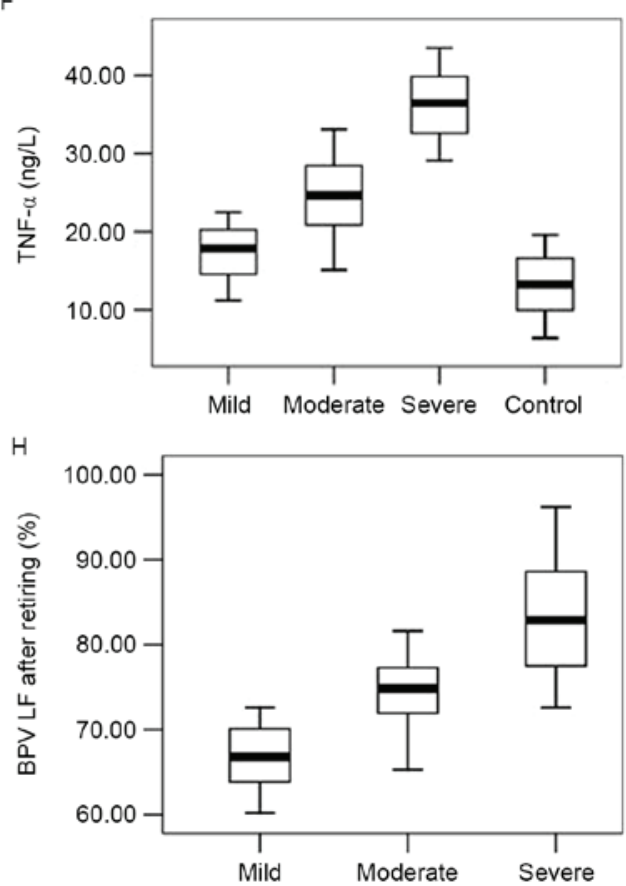

Figure 1. Physiological measurements among patients with OSAHS and controls. (A) AHI levels; (B) LSaO ; (C) carotid IMT; (D) plasma ET-1; (E) NO levels; (F) TNF- $\alpha$ levels; (G) BPV LF before retiring; and (H) BPV LF after retiring. OSAHS, obstructive sleep apnea hypopnea syndrome; AHI, apnea-hypopnea index; $\mathrm{LSaO}_{2}$, lowest blood oxygen saturation; IMT, intima media thickness; ET-1, endothelin-1; NO, nitric oxide; TNF- $\alpha$, tumor necrosis factor- $\alpha$; BPV LF, low frequency components of blood pressure variability. 
Table II. Multiple linear regression analysis of the influence of carotid IMT on OSAHS patients.

\begin{tabular}{|c|c|c|c|c|c|}
\hline \multirow[b]{2}{*}{ Variables } & \multicolumn{2}{|c|}{$\begin{array}{l}\text { Unstandardized } \\
\text { coefficients }\end{array}$} & \multirow{2}{*}{$\frac{\begin{array}{c}\text { Standardized } \\
\text { coefficients }\end{array}}{\text { Beta }}$} & \multirow[b]{2}{*}{$\mathrm{t}$} & \multirow[b]{2}{*}{ P-value } \\
\hline & B & SE & & & \\
\hline (Constant) & 0.999 & 0.473 & & 2.112 & 0.037 \\
\hline AHI & 0.009 & 0.003 & 0.700 & 3.490 & 0.001 \\
\hline $\mathrm{LSaO}_{2}$ & -0.010 & 0.005 & -0.536 & -2.180 & 0.031 \\
\hline ET-1 & 0.175 & 0.051 & 0.489 & 3.454 & 0.001 \\
\hline NO & -0.033 & 0.005 & -1.673 & -6.734 & 0.000 \\
\hline TNF- $\alpha$ & 0.000 & 0.001 & 0.036 & 0.350 & 0.727 \\
\hline BPV LF before retiring & 0.024 & 0.003 & 1.213 & 7.202 & $<0.001$ \\
\hline BPV LF after retiring & 0.007 & 0.004 & 0.305 & 1.747 & 0.083 \\
\hline
\end{tabular}

SE, standard error; IMT, intima media thickness; OSAHS, obstructive sleep apnea hypopnea syndrome; $\mathrm{AHI}$, apnea-hypopnea index; $\mathrm{LSaO}_{2}$, lowest blood oxygen saturation; ET-1, endothelin-1; NO, nitric oxide; TNF- $\alpha$, tumor necrosis factor- $\alpha$; BPV LF, low frequency components of blood pressure variability.
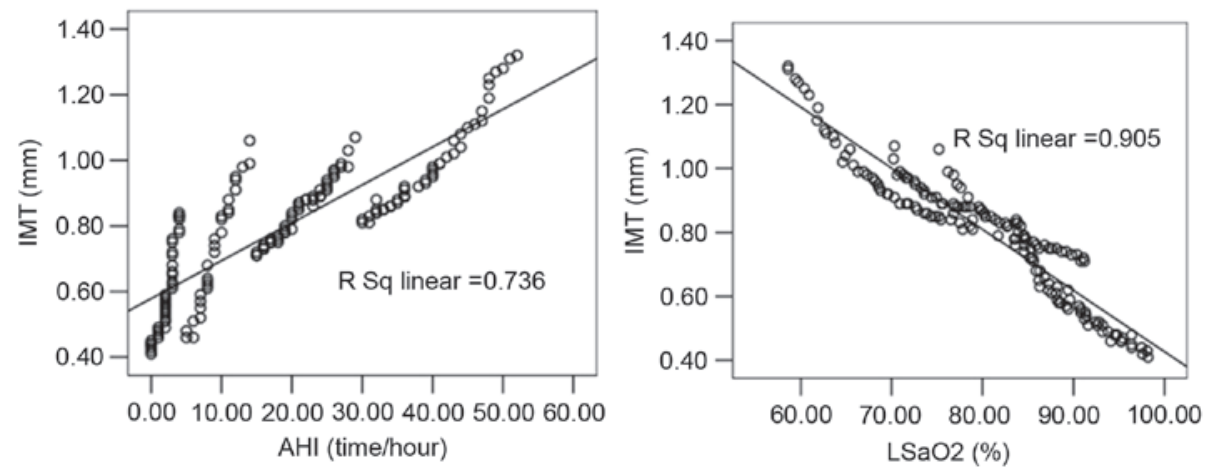

Figure 2. Correlation of carotid IMT with AHI and $\mathrm{LSaO}_{2}$ in patients with OSAHS. IMT, intima media thickness; $\mathrm{AHI}$, apnea-hypopnea index; $\mathrm{LSaO}_{2}$, lowest blood oxygen saturation; OSAHS, obstructive sleep apnea hypopnea syndrome.

factor analysis of variance was used to assess differences between multiple groups while the least significant difference method was used for pair-wise comparison and the paired Student's t-test was used to assess differences within the same group before and after treatment. Pearson's correlation and multiple linear regression analysis were used for correlation analysis. $\mathrm{P}<0.05$ was considered to indicate a statistically significant difference.

\section{Results}

AHI, IMT, ET-1, TNF- $\alpha, \mathrm{LSaO}_{2}$, and NO are affected by OSAHS. Numerous physiological parameters were significantly altered in patients with OSAHS, compared with healthy controls prior to the start of CPAP treatment. AHI, IMT, plasma ET-1, and plasma TNF- $\alpha$ levels of the patients with OSAHS were significantly higher than those in control patients $(\mathrm{P}<0.05)$, while $\mathrm{LSaO}_{2}$ and plasma $\mathrm{NO}$ levels were significantly lower in OSAHS patients than those in the control group ( $\mathrm{P}<0.05$; Fig. 1). All apnea patients' BPV LF significantly increased after sleep $(\mathrm{P}<0.05)$. The degree to which these parameters were affected was associated with the severity of the patients' condition.
Carotid arteriosclerosis is correlated with multiple physiological parameters in patients with OSAHS. Linear correlation analysis indicated that the carotid IMT of patients in the case groups was significantly correlated with AHI $(\mathrm{r}=0.858), \mathrm{LSaO}_{2}(\mathrm{r}=-0.952)$, plasma ET-1 $(\mathrm{r}=0.901)$, plasma NO $(r=-0.944)$, plasma TNF- $\alpha(r=0.918)$, BPV LF before sleep $(r=0.956)$, and BPV LF after sleep ( $r=0.924$; Figs. 2-4). Multiple linear regression analysis with the patient's carotid IMT as the dependent variable and the physiological parameters as independent variables demonstrated that IMT was significantly correlated with AHI (standardized regression coefficient, 0.700), $\mathrm{LSaO}_{2}$ (standardized regression coefficient, -0.536), plasma ET-1 (standardized regression coefficient, 0.489), plasma NO (standardized regression coefficient, -1.673), and BPV LV before sleep (standardized regression coefficient, 1.213; $\mathrm{P}<0.05)$, as shown in Table II. No significant correlation was detected between IMT and TNF- $\alpha$ or BPV LF after sleep.

CPAP treatment improves indicators of cardiovascular health in patients with moderate and severe OSAHS. After six months of CPAP treatment, the levels of AHI, IMT, ET-1, TNF- $\alpha$, BPV LF before and after retiring were decreased, 

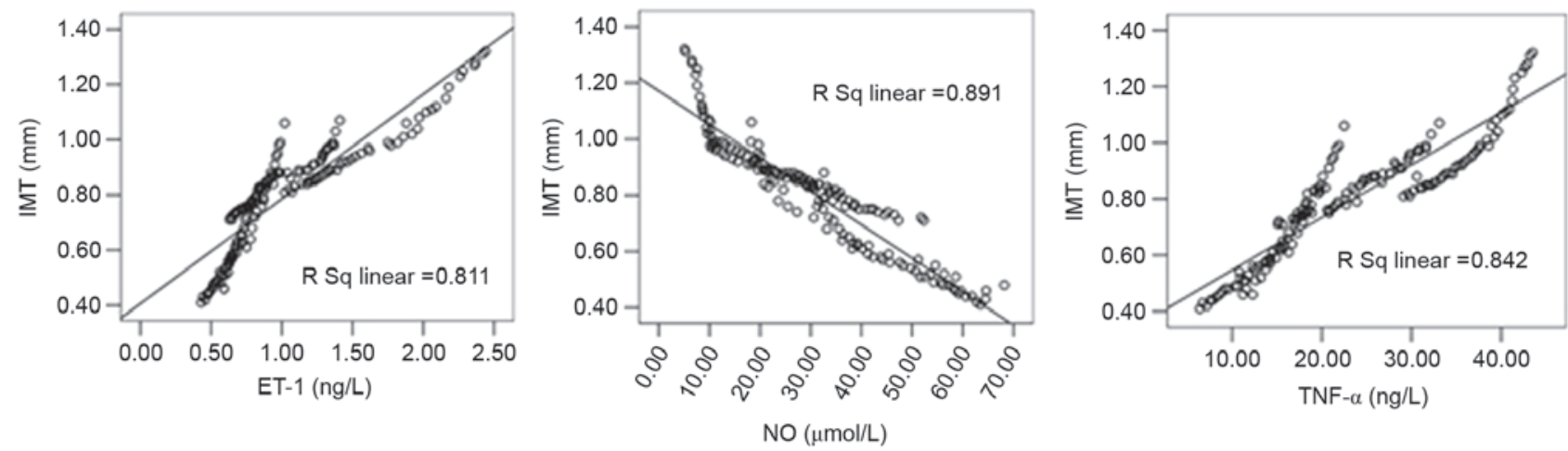

Figure 3. Correlation of carotid IMT with plasma ET-1, plasma NO, and TNF- $\alpha$ in patients with OSAHS. IMT, intima media thickness; ET-1, endothelin-1; NO, nitric oxide; TNF- $\alpha$, tumor necrosis factor- $\alpha$; OSAHS, obstructive sleep apnea hypopnea syndrome.
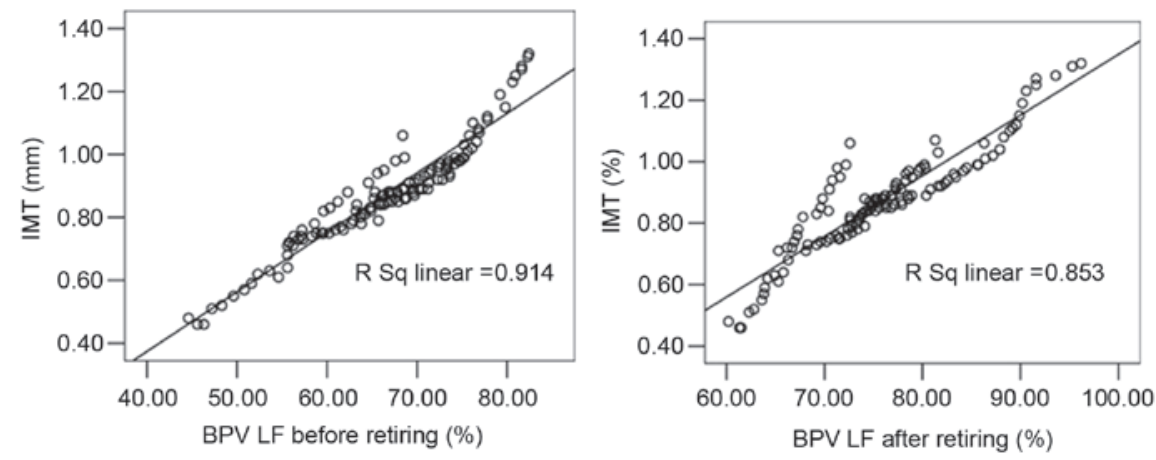

Figure 4. Correlation of carotid IMT with BPV LF before and after sleep in patients with OSAHS. IMT, intima media thickness; BPV LF, low frequency components of blood pressure variability; OSAHS, obstructive sleep apnea hypopnea syndrome.
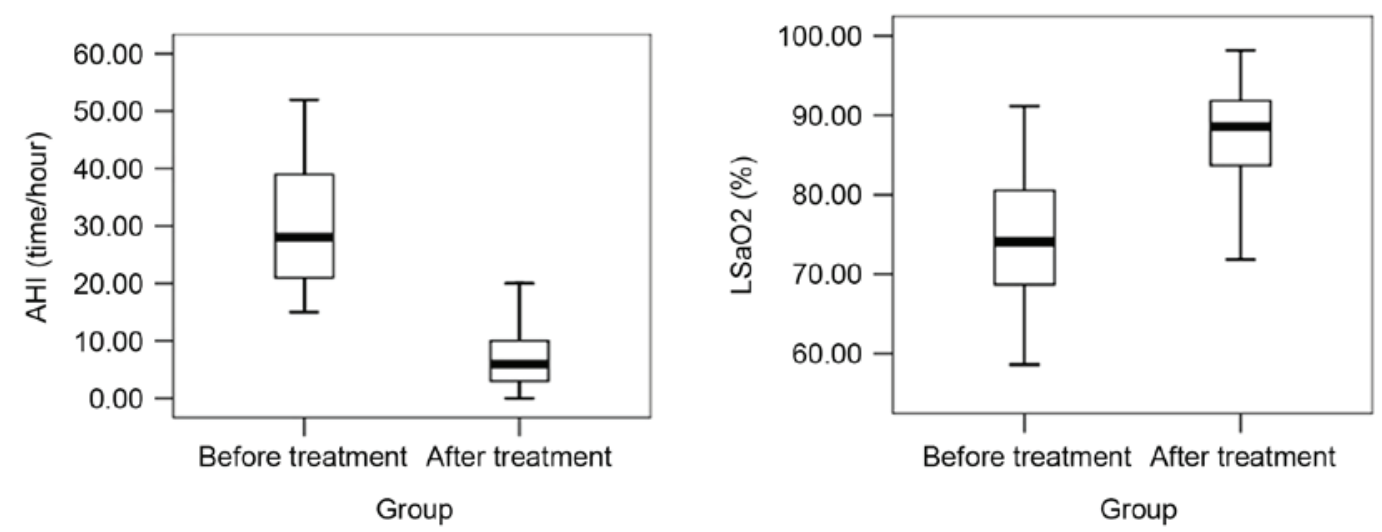

Figure 5. Changes in $\mathrm{AHI}$ and $\mathrm{LSaO}_{2}$ in patients with moderate and severe OSAHS after six months of $\mathrm{CPAP}$ treatment. $\mathrm{P}<0.01 ; \mathrm{t}_{\mathrm{AHI}}=27.436 ; \mathrm{t}_{\mathrm{LSaO}}=-22.263$. AHI, apnea-hypopnea index; $\mathrm{LSaO}_{2}$, lowest blood oxygen saturation; OSAHS, obstructive sleep apnea hypopnea syndrome; CPAP, continuous positive airway pressure.

while those of $\mathrm{LSaO}_{2}$ and $\mathrm{NO}$ were increased $(\mathrm{P}<0.05$; Figs. 5-7). In brief, all 92 patients following 6 months of CPAP treatment exhibited improvements in the measurements of the physiological parameters observed in this study.

\section{Discussion}

The results of the present study demonstrated that AHI, IMT, plasma ET-1, plasma TNF- $\alpha$ levels, and blood pressure measurements of patients with OSAHS were higher than those of the control group, whereas $\mathrm{LSaO}_{2}$ and plasma NO levels were lower in the control group. These differences became more pronounced as the severity of OSAHS increased. Furthermore, carotid IMT of patients with OSAHS was significantly correlated with $\mathrm{AHI}, \mathrm{LSaO}_{2}$, plasma ET-1, plasma NO, plasma TNF- $\alpha$, BPV LF before sleep, and BPV LF after sleep. These findings are consistent with those of previous studies that noted the correlation between sleep apnea and various physiological and plasma markers of cardiovascular function (4,7-10,14-16). 

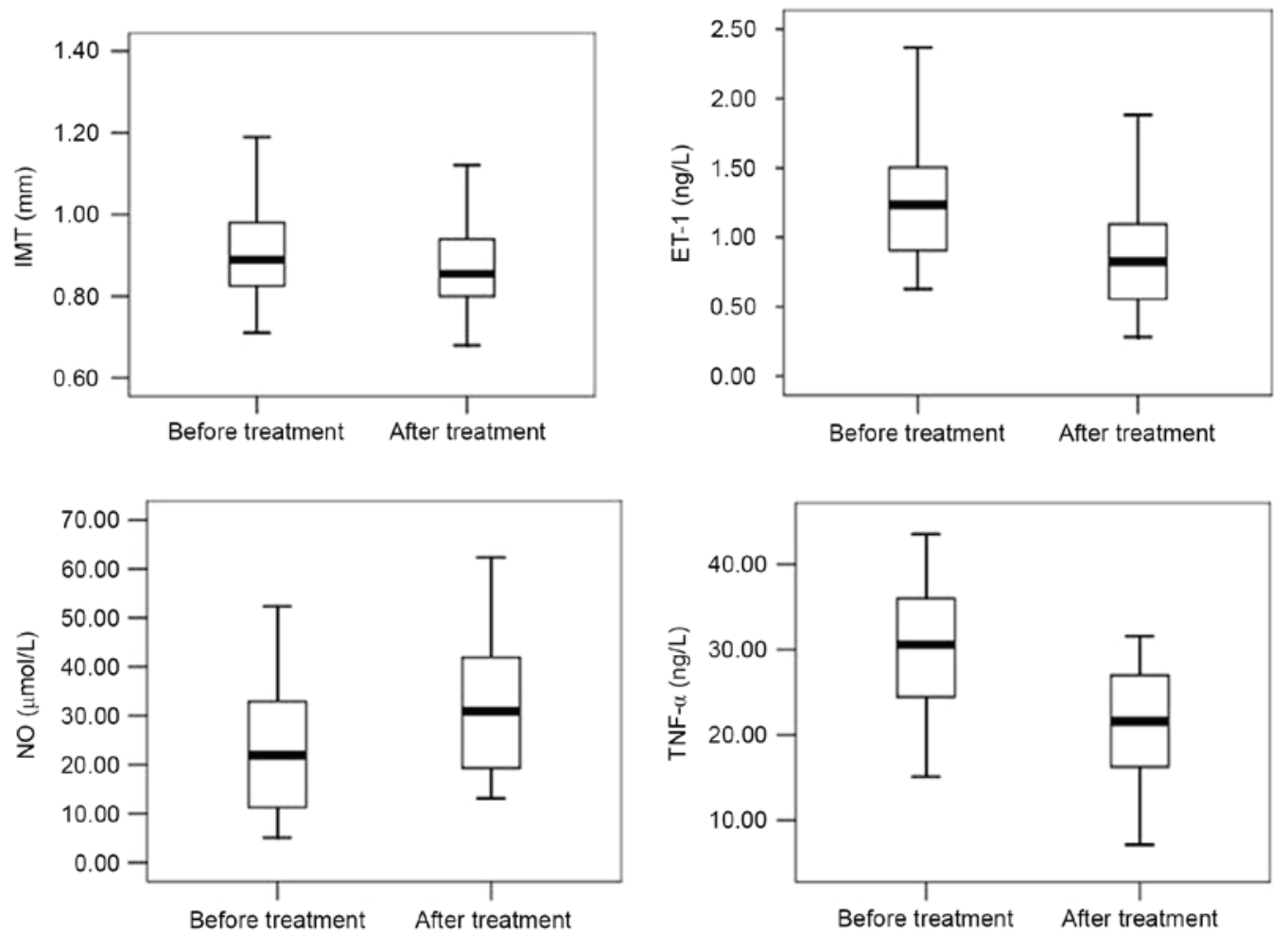

Figure 6. Changes in carotid IMT, plasma ET-1, NO and TNF- $\alpha$ levels of patiwnts with moderate and severe OSAHS after six months of CPAP treatment. $\mathrm{P}<0.01 ; \mathrm{t}_{\mathrm{IMT}}=28.683 ; \mathrm{t}_{\mathrm{ET}-1}=92.553 ; \mathrm{t}_{\mathrm{NO}}=-137.649 ; \mathrm{t}_{\mathrm{TNF}}{ }^{-} \alpha=88.407$. IMT, intima media thickness; ET-1, endothelin-1; NO, nitric oxide; TNF- $\alpha$, tumor necrosis factor- $\alpha$; OSAHS, obstructive sleep apnea hypopnea syndrome; CPAP, continuous positive airway pressure.
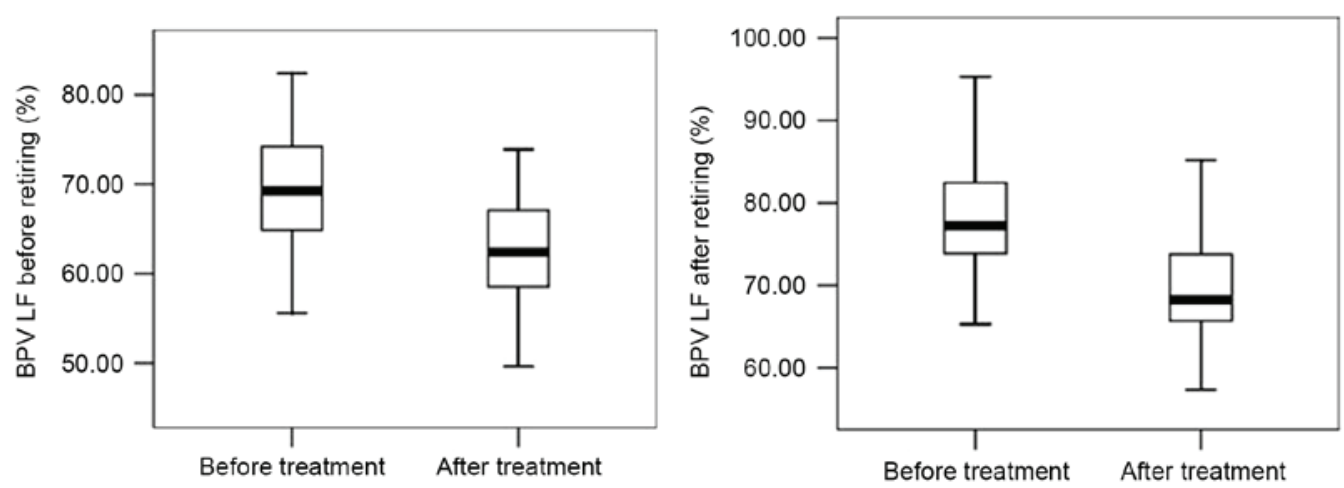

Figure 7. Changes in BPV LF levels before and after sleep in patients with moderate and severe OSAHS after six months of CPAP treatment. P $<0.01$; $\mathrm{t}=$ BPV LF before retiring $=90.417 ; \mathrm{t}_{\mathrm{BPV} \text { LF after retiring }}=95.436$. BPV LF, low frequency components of blood pressure variability; OSAHS, obstructive sleep apnea hypopnea syndrome; CPAP, continuous positive airway pressure.

Following 6 months of CPAP treatment, all of the physiological variables observed in our study were significantly improved among the patients with OSAHS. The results corroborate the findings of earlier studies, which reported benefits to these variables separately following CPAP treatment $(9,11-13,17,18)$. Therefore, the present findings support the hypothesis that CPAP treatment as an effective strategy for improving the pathological status of patients with moderate and severe OSAHS, whilst lowering the risk of cardiovascular disease.

The present study has some notable strengths. The inclusion of a follow-up period enabled assessment of the short-term benefits to treatment. Furthermore, the study included a sufficient sample size to stratify the patient population by severity. However, this study also has some limitations. Although the sample size was able to detect statistical differences, randomized controlled trials with larger samples are required to validate the utility of CPAP in treating OSAHS and reducing the risk of cardiovascular disease. A longer follow-up period would also enable an improved understanding of the benefits of treatment for chronic OSAHS in improving cardiovascular risks. A more comprehensive measure of cardiovascular health markers may help develop a clear picture of the effect of CPAP treatment on cardiovascular health, as well as guiding future work to understand the underlying mechanisms. 


\section{References}

1. Chen X, Sun J, Yuan W, Yuan W and Li J: OSAHS obstructive plane localization: Comparative study between ag200 and friedman classification. Int J Clin Exp Med 8: 2240-2246, 2015.

2. Lombardi C, Musicco E, Bettoncelli G, Milanese M, Senna G, Braido F and Canonica GW: The perception of Obstructive Sleep Apnoea/Hypopnoea Syndrome (OSAHS) among Italian general practitioners. Clin Mol Allergy 13: 4, 2015.

3. Chen Y, Li Y, Jiang Q, Xu X, Zhang X, Simayi Z and Ye H: Analysis of early kidney injury-related factors in patients with hypertension and Obstructive Sleep Apnea Hypopnea Syndrome (OSAHS). Arch Iran Med 18: 827-833, 2015.

4. Chen H, Hu K, Zhu J, Xianyu Y, Cao X, Kang J, He J, Zhao P and Mei Y: Polymorphisms of the 5-hydroxytryptamine 2A/2C receptor genes and 5-hydroxytryptamine transporter gene in Chinese patients with OSAHS. Sleep Breath 17: 1241-1248, 2013.

5. Vijayan VK: Morbidities associated with obstructive sleep apnea. Expert Rev Respir Med 6: 557-566, 2012.

6. Chen S, Shi S, Xia Y, Liu F, Chen D, Zhu M, Li M and Zheng H: Changes in sleep characteristics and airway obstruction in OSAHS patients with multi-level obstruction following simple UPPP, UPPP-GA, or UPPP-TBA: A prospective, single-center, parallel group study. ORL J Otorhinolaryngol Relat Spec 76: 179-188, 2014.

7. Shi S, Xia Y, Zhu M, Wei K, Liu F, Chen D, Chen S and Zheng H: Characterization of upper airway obstruction by fiber-optic nasolaryngoscopy and MRI in preoperative OSAHS patients. ORL J Otorhinolaryngol Relat Spec 76: 321-328, 2014.

8. Chalghoum A, Noichri Y, Dandana A, Azaiez S, Baudin B, Jeridi G, Miled A and Ferchichi S: Relationship between the $\mathrm{A}(8002) \mathrm{G}$ intronic polymorphism of pre-pro-endothelin-1 gene and the endothelin-1 concentration among Tunisian coronary patients. BMC Cardiovasc Disord 15: 152, 2015.

9. Anunciato IF, Lobo RR, Coelho EB, Verri WA Jr, Eckeli AL, Evora PR, Nobre F, Moriguti JC, Ferriolli E and Lima NK: Big endothelin-1 and nitric oxide in hypertensive elderly patients with and without obstructive sleep apnea-hypopnea syndrome. Arq Bras Cardiol 101: 344-351, 2013.
10. Sukhovershin RA, Yepuri $G$ and Ghebremariam YT: Endothelium-derived nitric oxide as an antiatherogenic mechanism: Implications for therapy. Methodist Debakey Cardiovasc J 11: 166-171, 2015.

11. Reddy YS, Kiranmayi VS, Bitla AR, Krishna GS, Rao PV and Sivakumar V: Nitric oxide status in patients with chronic kidney disease. Indian J Nephrol 25: 287-291, 2015.

12. Zhong A, Xiong X, Xu H and Shi M: An updated meta-analysis of the association between tumor necrosis factor- $\alpha-308 \mathrm{G} / \mathrm{A}$ polymorphism and obstructive sleep apnea-hypopnea syndrome. PLoS One 9: e106270, 2014.

13. Almpanidou P, Hadjigeorgiou G, Gourgoulianis K and Papadimitriou A: Association of tumor necrosis factor- $\alpha$ gene polymorphism (-308) and obstructive sleep apnea-hypopnea syndrome. Hippokratia 16: 217-220, 2012.

14. Chenniappan M: Blood pressure variability: Assessment, prognostic significance and management. J Assoc Physicians India 63: 47-53, 2015.

15. An S, Bao M, Wang Y, Li Z, Zhang W, Chen S, Li J, Yang X, Wu S and Cai J. Relationship between cardiovascular health score and year-to-year blood pressure variability in China: A prospective cohort study. BMJ Open 5: e008730, 2015.

16. Brockmann PE: Cardiovascular consequences in children with obstructive sleep apnea: Is it possible to predict them? Sleep 38: 1343-1344, 2015.

17. Ewen S, Dörr O, Ukena C, Linz D, Cremers B, Laufs U, Hamm C, Nef H, Bauer A, Mancia G, et al. Blood pressure variability after catheter-based renal sympathetic denervation in patients with resistant hypertension. J Hypertens 33: 2512-2518, 2015.

18. Li J, Chen X and Sun J: Is the grading system of the severity of the OSAHS used presently rational or not? From the view of incidence of hypertension in different severity groups. Eur Arch Otorhinolaryngol 271: 2561-2564, 2014.

19. Ben Saad H, Ben Hassen I, Ghannouchi I, Latiri I, Rouatbi S, Escourrou P, Ben Salem H, Benzarti M and Abdelghani A: 6-Min walk-test data in severe obstructive-sleep-apnea-hypopnea-syn drome (OSAHS) under continuous- positive- airway- pressure (CPAP) treatment. Respir Med 109: 642-655, 2015.

20. Chinese Medical Association Respiratory Diseases Branch: OSAHS Diagnosis and Treatment (Modified Version). Chinese J Tuberculosis Respiratory Dis 35: 9-12, 2012 (In Chinese). 